\title{
Limits on iron-dominated fallback disk in SN 1987A
}

\author{
K. Werner, T. Nagel and T. Rauch \\ Institute for Astronomy and Astrophysics, University of Tübingen, 72076 Tübingen, Germany
}

\begin{abstract}
The non-detection of a point source in SN 1987A imposes an upper limit for the optical luminosity of $L_{\mathrm{opt}} \approx 2 \mathrm{~L}_{\odot}$. This limits the size of a possible fallback disk around the stellar remnant. Assuming a steady-state thin disk with blackbody emission requires a disk smaller than $100000 \mathrm{~km}$ if the accretion rate is at $30 \%$ of the Eddington rate [6]. We have performed detailed non-LTE radiation transfer calculations to model the disk spectrum more realistically. It turns out that the observational limit on the disk extension becomes even tighter, namely $70000 \mathrm{~km}$.
\end{abstract}

Keywords: Supernovae - Accretion and accretion disks

PACS: $97.60 . \mathrm{Bw}, 97.10 . \mathrm{Gz}$

\section{INTRODUCTION}

To date there is no direct evidence for a compact object at the center of the SN 1987A remnant. The initial neutrino burst is still the only evidence for the formation of a compact object which could be either a neutron star or a black hole. The non-detection of a point source in HST images imposes a tight upper limit to its optical luminosity, which cannot exceed about $2 \mathrm{~L} \odot$ [6].

This allows to draw tight limits on the size of a possible supernova fallback-disk around the compact object. Assuming steady-state thin-disk accretion the optical/UV luminosity depends on the mass-accretion rate and outer disk radius. For example, it was concluded that the observed optical flux limit requires a small disk, no larger than $10^{5} \mathrm{~km}$, with an accretion rate of no more than $30 \%$ of the Eddington accretion rate [6]. This result was obtained assuming a steady-state Shakura-Sunyaev disk [16] composed of concentric rings emitting blackbody spectra. It is our aim to see how the limits on the fallback disk are affected by the blackbody assumption and to this end we compute more realistic disk spectra by detailed radiation-transfer calculations.

Aside from the particular case of SN 1987A, observational proofs for the existence of SN-fallback disks around pulsars are still debated. The X-ray luminosities of anomalous X-ray pulsars (AXPs), which are slowly rotating $\left(P_{\text {rot }}=5-12 \mathrm{~s}\right)$, young $(\leq 100000 \mathrm{yr})$, isolated neutron stars, are generally assumed to be powered by magnetic energy [20]. As an alternative explanation the X-ray emission was attributed to accretion from a disk that is made up of SN-fallback material $[1,2,14]$.

The fallback-disk model, however, has difficulties to explain IR/optical emission properties of AXPs. When compared with disk models, the faint IR/optical flux suggests that any disk around AXPs must be very compact (e.g. [15, 7]). The discovery of optical pulsations in the AXP 4U 0142+61 which have the same period like the X-ray pulsations [8] appears to be a strong argument against the disk model. It was argued 
that reprocessing of the pulsed neutron star X-ray emission in the disk cannot explain the high optical pulsed fraction, because disk radiation would be dominated by viscous dissipation and not by reprocessed neutron star irradiation [8]. On the other hand, it was shown that these optical pulsations can be explained either by the magnetar outer gap model or by the disk-star dynamo model [5]. Therefore, the observation of optical pulsations is not an argument against the disk model.

The recent discovery of mid-IR emission from this AXP [17], however, has strongly rekindled the interest in studies of fallback-disk emission properties. While this midIR emission is attributed to a cool, passive (X-ray irradiated) dust debris disk [17], all optical and near-IR flux measurements can be explained with a model for an active, dissipating gas disk [4].

Coming back to SN 1987A, the presence of a fallback disk around its stellar remnant has been invoked in order to explain its observed lightcurve which deviates from the theoretical one for pure radioactive decay [11].

\section{MODEL ASSUMPTIONS}

We employ our computer code ACDC [12], that calculates disk spectra under the following assumptions. The radial disk structure is calculated assuming a stationary, Keplerian, geometrically thin $\alpha$-disk [16]. As pointed out in [10], for a comparison with observational data one probably has to use a more elaborate model, because near the outer disk edge the viscous dissipation and hence the surface mass density decline stronger with increasing radius than in an $\alpha$-disk. However, the purpose of the present paper is to look for differential effects of various assumptions. Qualitatively, these effects can be expected to be independent of the detailed radial disk structure. In any case, it would be no problem to carry out the computations presented here with different radial structures.

The $\alpha$-disk model is fixed by four global input parameters: Stellar mass $M_{\star}$ and radius $R_{\star}$ of the accretor, mass accretion rate $\dot{M}$, and the viscosity parameter $\alpha$. For numerical treatment the disk is represented by a number of concentric rings. For each ring with radius $R$ our code calculates the detailed vertical structure, assuming a plane-parallel radiating slab.

A particular disk ring with radius $R$ is characterized by the following two parameters, which follow from the global disk parameters introduced above. The first parameter measures the dissipated and then radiated energy. It can be expressed in terms of an effective temperature $T_{\text {eff }}$, and the second parameter is the half surface mass density $\Sigma$ of the disk ring:

$$
T_{\mathrm{eff}}^{4}(R)=\left[1-\left(R_{\star} / R\right)^{1 / 2}\right] 3 G M_{\star} \dot{M} / 8 \sigma \pi R^{3} \quad \Sigma(R)=\left[1-\left(R_{\star} / R\right)^{1 / 2}\right] \dot{M} / 3 \pi \bar{w} .
$$

$\sigma$ and $G$ are the Stefan-Boltzmann and gravitational constants, respectively. $\bar{w}$ is the depth mean of viscosity $w(z)$, where $z$ is the height above the disk mid-plane. The viscosity is given by the standard $\alpha$-parametrization as a function of the total (i.e. gas plus radiation) pressure, but numerous other modified versions are used in the literature. We use a formulation involving the Reynolds number $R e$, as proposed by [9] and we set $R e=15000$ which corresponds to $\alpha \approx 0.01$. 

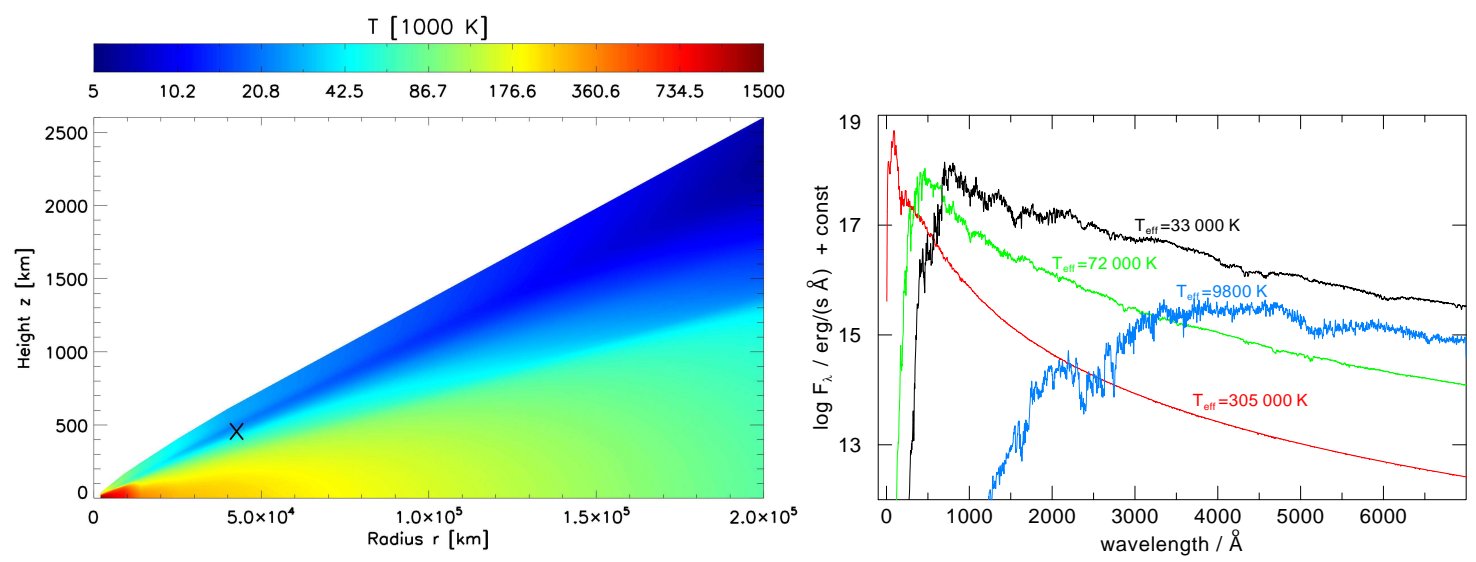

FIGURE 1. Left: This cut perpendicular to the midplane shows the temperature structure of the disk. Note that the vertical scale (height above the midplane) is expanded. The cross marks the depth at $R=40000 \mathrm{~km}$ where $\tau_{\text {Ross }}=1$. Right: Relative contribution of four individual disk rings to the total disk flux. The flux from the ring at $R=40000 \mathrm{~km}$ with $T_{\text {eff }}=33000 \mathrm{~K}$ dominates the total disk spectrum at UV/optical wavelengths.

For the results presented here we selected the following parameters. The NS mass is $1.4 \mathrm{M}_{\odot}$. The radii of the inner and outer disk edges are 2000 and $200000 \mathrm{~km}$, respectively. The disk is represented by nine rings or, more precisely, radial grid points. The accretion rate is $\dot{M}=3 \cdot 10^{-9} \mathrm{M}_{\odot} / \mathrm{yr}$, corresponding to $30 \%$ of the Eddington rate. A detailed discussion of the model characteristics can be found in [19].

The vertical structure of each disk ring is determined from the simultaneous solution of the radiation transfer equations plus the structure equations. The latter invoke radiative and hydrostatic equilibrium plus charge conservation and also consist of the NLTE rate equations for the atomic population densities. The solution of this set of highly non-linear integro-differential equations is performed using the Accelerated Lambda Iteration technique [18]. Our code allows for the irradiation of the disk by the central source, however, the results presented here are computed with zero incident intensity.

The radiation-transfer equations plus vertical structure equations are solved like in the stellar atmosphere case, but accounting for two basic differences. First, the gravity (entering the hydrostatic equation for the total, i.e. gas plus radiation, pressure) is not constant with depth, but increases with $z$. The gravity is the vertical component of the gravitational acceleration exerted by the central object (self-gravitation of the disk is negligible). Second, the energy equation for radiative equilibrium balances the dissipated mechanical energy and the net radiative losses.

For each atomic level $i$ the NLTE rate equation describes the equilibrium of rates into and rates out of this level and, thus, determine the occupation numbers $n_{i}$. The rate coefficients have radiative and electron collisional components. The blanketing by millions of lines from iron arising from transitions between some $10^{5}$ levels can only be attacked with the help of statistical methods [3]. We have created a detailed iron model atom for the NLTE calculations. It comprises the lowest eleven ionisation stages and a total number of more than 3 million lines.

The composition of the fallback material in the disk is not exactly known. It depends 
on the amount of mass that goes into the disk. A disk with a small mass $\left(\lesssim 0.001 \mathrm{M}_{\odot}\right)$ will be composed of Si-burning ash [10]. For simplicity, the results presented here are obtained by assuming a pure-Fe composition. Tests show that the emergent spectrum is insensitive against the exact composition as long as $\mathrm{Fe}$ is the dominant species.

Having calculated the vertical structures and spectra of the individual disk rings, the ring spectra are integrated taking into account Keplerian rotation.

\section{RESULTS}

The left panel in Fig. 1 displays the temperature structure of the disk. The temperature varies between 1.5 million $\mathrm{K}$ in the midplane at the inner disk edge down to $6000 \mathrm{~K}$ in the upper layers at the outer disk edge.

Which disk regions contribute to the total disk spectrum and to what extent? In the right panel of Fig. 1 we plot the emergent astrophysical flux from the area of four disk rings, i.e., the computed flux per $\mathrm{cm}^{2}$ is weighted with the ring area. The spectral flux distribution of the innermost ring with $T_{\text {eff }}=305000 \mathrm{~K}$ has its peak value in the soft X-ray region. The contribution of this disk region to the optical/UV spectrum is negligible. Cutting off the disk at this inner radius $(R=2000 \mathrm{~km})$, therefore, is justified if this spectral range is of interest. The disk region that is dominating the UV/optical flux is represented by the ring at $R=40000 \mathrm{~km}$ with $T_{\mathrm{eff}}=33000 \mathrm{~K}$. Its spectrum is dominated by strong blends of the numerous iron lines. Further out in the disk $T_{\text {eff }}$ decreases and the flux contribution to the UV/optical spectrum declines, too. Our outermost ring has $T_{\text {eff }}=9800 \mathrm{~K}$, its flux maximum is at $\lambda=4000 \AA$ and it is fainter than the inner neighbor ring over the whole spectral range. Cutting off the disk at this outer radius $(R=200000 \mathrm{~km})$ therefore does not affect the UV/optical spectral region.

Our model spectra show distinct limb-darkening effects. The situation is similar to the stellar atmosphere case (center-to-limb variation of the specific intensity). Looking face-on we see into deeper and hotter (and thus "brighter") layers of the disk when compared to a more edge-on view. In Fig. 2 we compare the specific intensity emitted at $R=40000 \mathrm{~km}$ (per unit area) for a high and a low inclination angle. Overall, the "edge-on" spectrum is roughly a factor of two fainter than the "face-on" spectrum in the optical region. The difference increases towards the UV. We conclude that limb darkening effects are important when disk dimensions are to be estimated from magnitude measurements.

We now compare the intensities with a blackbody spectrum (Fig. 2). Depending on the wavelength band, the blackbody over- or underestimates the "real" spectrum up to a factor of two in the optical and a factor of four in the UV. The upper limit of $R=10^{5} \mathrm{~km}$ for the SN 1987A disk extension at this particular accretion rate was derived from an HST observation performed with the F330W filter. Its passband is indicated in Fig.2. Assuming a disk inclination that coincides with that of the observed inner equatorial ring around SN 1987A $\left(i=43^{\circ},[13]\right)$ one can see that the blackbody assumption systematically underestimates the flux in this wavelength region by about $20 \%$. This means that the observed flux limit together with our model imposes an even stronger limit on the disk extension. In order to reduce the disk luminosity in this wavelength region by $20 \%$ its extension must be reduced by about $30 \%$. Hence we find that the 


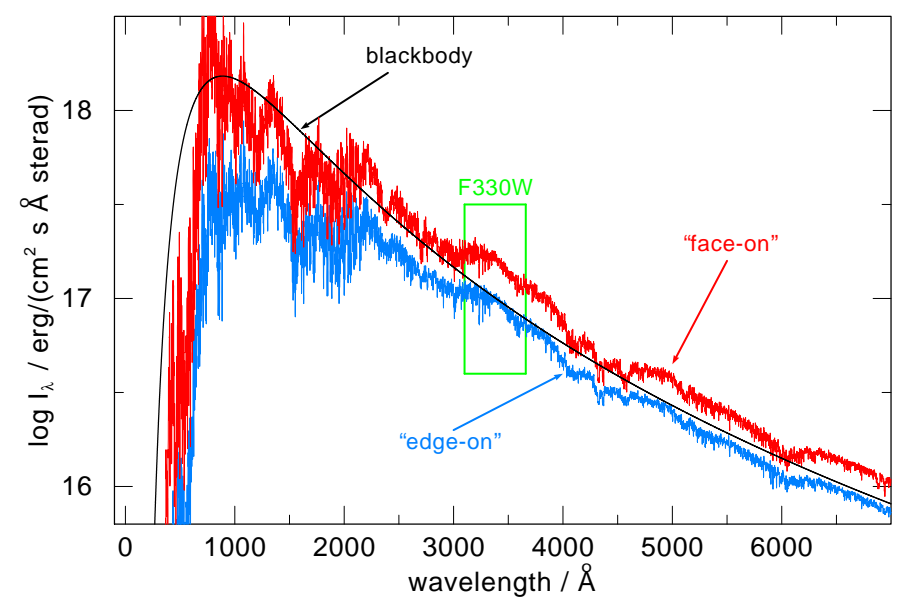

FIGURE 2. Effect of limb darkening: Specific intensity of the disk at $R=40000 \mathrm{~km}$ seen under inclination angles $87^{\circ}$ (i.e. almost edge-on) and $18^{\circ}$ (i.e. almost face-on). For comparison we also show a blackbody spectrum with $T=T_{\text {eff }}(R)=33000 \mathrm{~K}$. The rectangle indicates the passband of the HST/ACS filter F330W: The blackbody spectrum systematically underestimates the flux for intermediate inclinations.

upper limit for the disk extension must be reduced from $100000 \mathrm{~km}$ to $70000 \mathrm{~km}$.

Acknowledgement T.R. is supported by the German Ministry of Education and Research through DESY under grant 05 AC6VTB.

\section{REFERENCES}

1. Alpar, M. A. ApJ, 554, 1245 (2001)

2. Chatterjee, P., Hernquist, L., \& Narayan, R. ApJ, 534, 373 (2000)

3. Dreizler, S., \& Werner, K. A\&A, 278, 199 (1993)

4. Ertan, Ü., Erkut, M.H., Ekşi, K. Y., Alpar, M. A. ApJ, 657, 441 (2007)

5. Ertan, Ü., \& Cheng, K. S. ApJ, 605, 840 (2004)

6. Graves, G. J. M., Challis, P. M., Chevalier, R. A., et al. ApJ, 629, 944 (2005)

7. Israel, G. L., Rea, N., Mangano, V., et al. ApJ, 603, L97 (2004)

8. Kern, B., \& Martin, C. Nature, 417, 527 (2002)

9. Kriz, S., \& Hubeny, I. Bull. Astron. Inst. Czech., 37, 129 (1986)

10. Menou, K., Perna, R., \& Hernquist, L. ApJ, 559, 1032 (2001)

11. Meyer-Hofmeister, E. A\&A, 253, 459 (1992)

12. Nagel, T., Dreizler, S., Rauch, T., \& Werner, K. A\&A, 428, 109 (2004)

13. Panagia, N., Gilmozzi, R., Macchetto, F., Adorf, H.-M., \& Kirshner, R. P. ApJ, 380, L23 (1991)

14. van Paradijs, J., Taam, R. E., \& van den Heuvel, E. P. J. A\&A, 299, L41 (1995)

15. Perna, R., Hernquist, L., \& Narayan, R. ApJ, 541, 344 (2000)

16. Shakura N. I., \& Sunyaev R. A. A\&A, 24, 337 (1973)

17. Wang, Z., Chakrabarty, D., \& Kaplan, D. L. Nature, 440, 772 (2006)

18. Werner, K., Deetjen, J. L., Dreizler, S., et al. in: Hubeny, I., Mihalas, D., Werner, K. (ed.) Stellar Atmosphere Modeling, ASP Conf. Series, 288, 31 (2003)

19. Werner, K., Nagel, T., \& Rauch, T. Ap\&SS, in press, astro-ph/0608529 (2007)

20. Woods, P. M., \& Thompson, C. in: Lewin, W. H. G., van der Klis, M. (ed.) Compact Stellar X-Ray Sources, Cambridge University Press, p. 547 (2006) 